\title{
PAIN MODULATION PROFILE PRDICTS PATIENT DELAY IN SEEKING MEDICAL HELP IN ACUTE MYOCARDIAL INFARCTION
}

\author{
Michal Granot, Ph.D ${ }^{1}$ \\ ${ }^{1}$ The Laboratory of Clinical Neurophysiology, the Rappaport Faculty of Medicine, Technion - Israel Institute of \\ Technology, and Faculty of Social Welfare and Health Sciences, University of Haifa, Israel.
}

\section{Background and Goal of Study}

Rapid reperfusion is crucial to reduce mortality in patients with ST-elevation myocardial infarction (STEMI). Prehospital patient delay, defined as time from symptoms onset to the decision to seek medical attention, accounts for a large proportion of cases with delayed reperfusion. However, whether altered pain modulation processes ${ }^{1}$ affect less severe pain symptoms and consequently prolonger delay needs further illumination. We explored whether prehospital patient delay is affected by a reduction of perceived pain perception and pain modulation response.

\section{Materials and Methods}

Facilitatory and inhibitory pain modulation pathways assessed by psychophysical tests of temporal summation and conditioned pain modulation (CPM) as well as sensation and pain thresholds, magnitude estimation of supra-threshold stimulation. Pain recalls at the onset of chest pain were obtained in 67 STEMI hospitalized patients. The associations between these measures and chest pain intensity and duration of patient delay were explored.

\section{Results and Discussion}

Among all psychophysical pain measures only warm sensation threshold was independently associated with lower clinical chest pain intensity $(p=0.01$ ). Multivariable regression analysis $\left(R^{2}=0.449 ; P<0.0001\right)$ revealed an inverse independent association between chest pain intensity $(P<0.001)$ and patient delay whereas efficient CPM was positively associated with prolonged patient delay $(P=0.034)$. The electrocardiography-derived myocardial ischemic area at risk for necrosis was not associated with chest pain intensity or patient delay. Beyond the perceived chest pain intensity, patients who exhibit efficient response of the descending inhibition pathways have prolonged delay in seeking medical help after the onset of chest pain during acute coronary occlusion.

Conclusion - The findings emphasize the significant role of the individual pain modulation profile 4 and may suggest new venue to identify patients with susceptibility to experience less pain and less hazard signal and consequently delay in seeking medical help.

Relationship between chest pain intensity and the electrocardiography derived myocardial area at risk

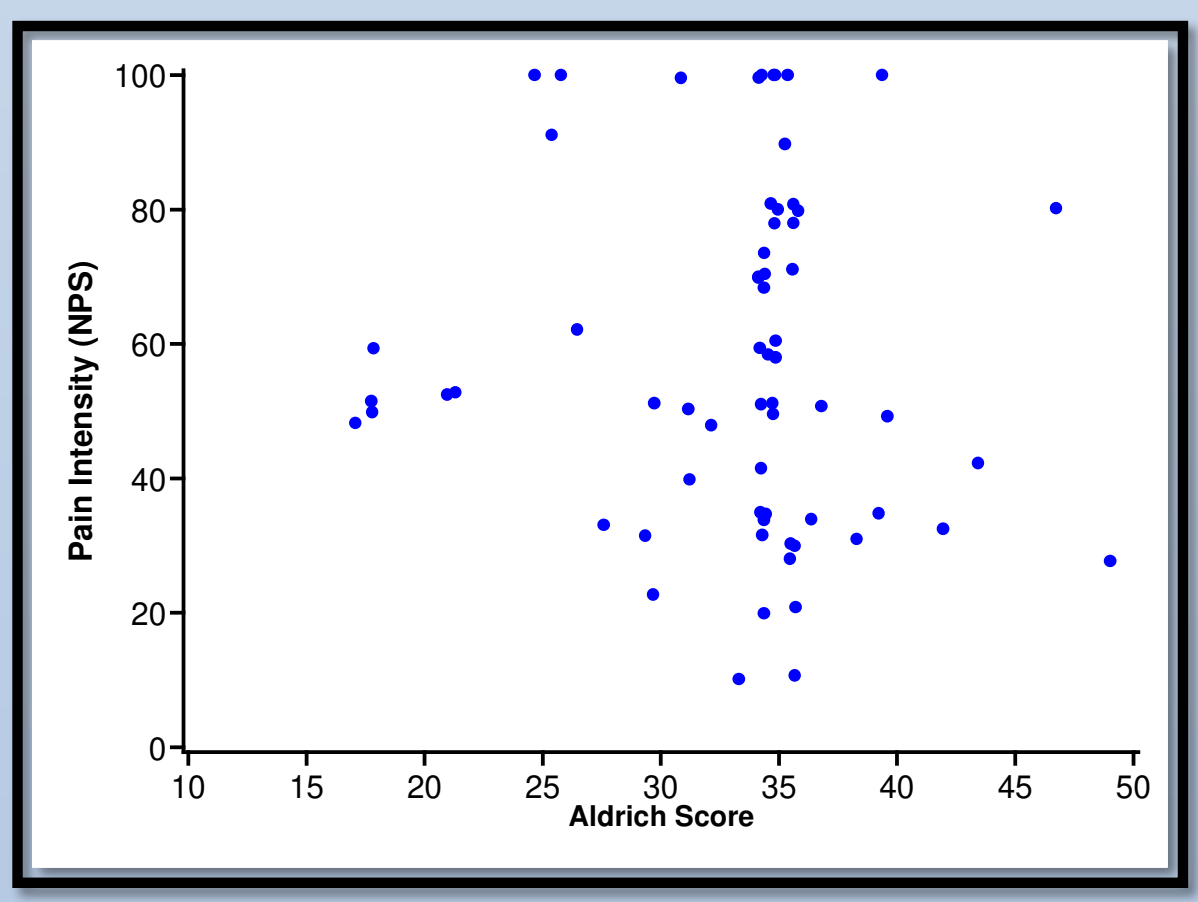

Association between perceived chest pain and duration of patient delay in seeking medical help

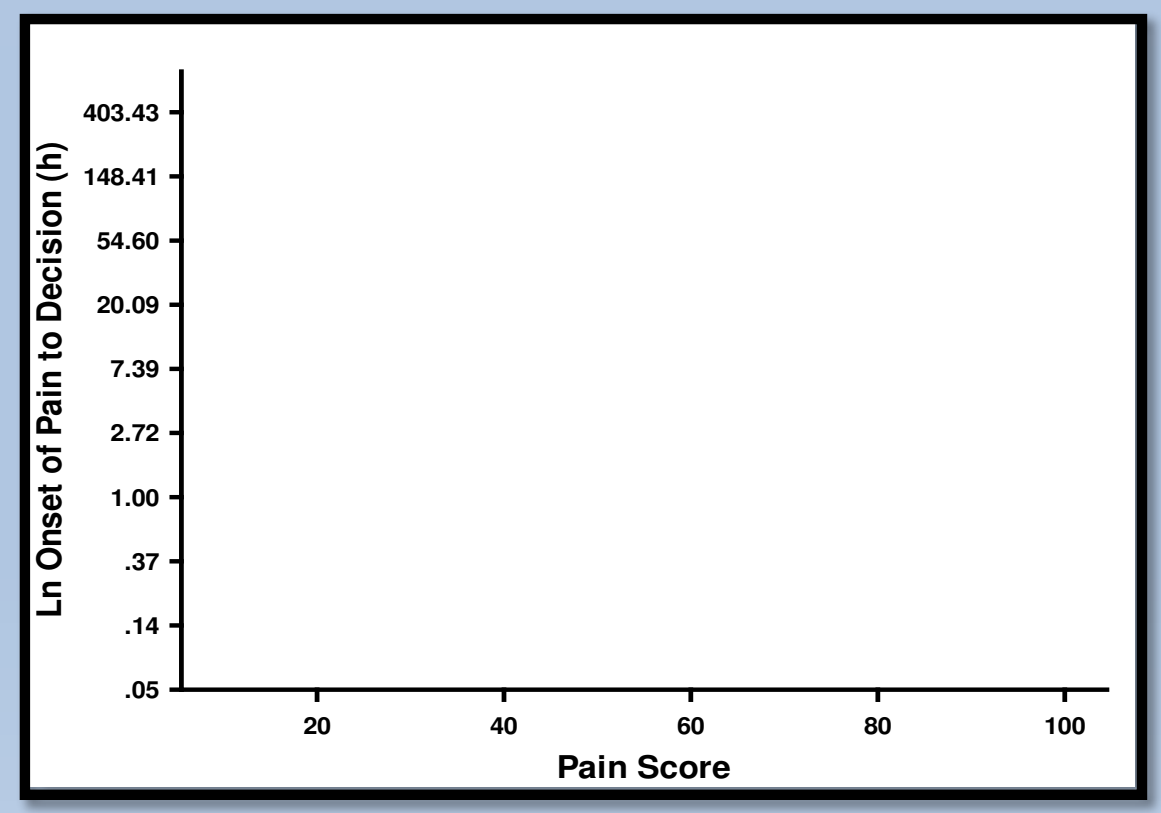

Greater efficacy of endogenous analgesia (CPM) affects patient delay in seeking medical help
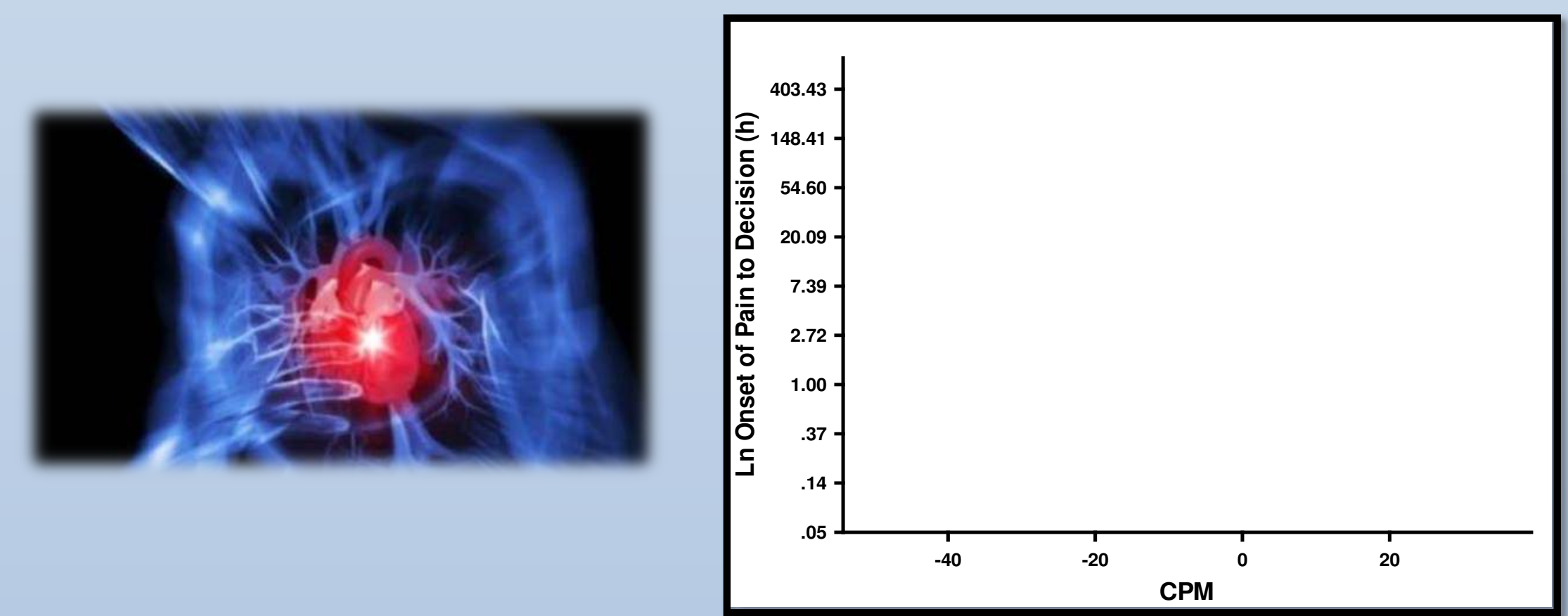

Multiple linear regression analysis depicting predictors of patient delay

\begin{tabular}{|c|c|c|c|c|c|c|}
\hline & Unadjusted & Adjusted & & & & \\
\hline Variable & $\begin{array}{l}\text { B Coefficient } \\
\text { (SE) }\end{array}$ & T & $\mathrm{p}$ & $\begin{array}{c}\text { B } \\
\text { Coefficient (SE) }\end{array}$ & T & $\mathrm{p}$ \\
\hline $\begin{array}{l}\text { Chest pain intensity } \\
\text { at onset of symptoms }\end{array}$ & -(0.01) 0.07 & -6.43 & $0.001>$ & $-(0.01) 0.06$ & -5.11 & $0.001>$ \\
\hline CPM & - 0.0202 (0.02 & -2.19 & 0.033 & $-(0.02) 0.04$ & -2.18 & 0.034 \\
\hline Radiating pain & (0.66) 1.99 & 3.04 & 0.003 & - & - & - \\
\hline $\begin{array}{l}\text { Mechanical sensation } \\
\text { threshold } \\
(\text { Ln) }\end{array}$ & (0.19) 0.47 & 2.44 & 0.018 & 0.33 (0.17) & 1.91 & 0.062 \\
\hline
\end{tabular}

References

${ }^{1}$ Granot M, Khoury R, Berger G, Krivoy N, Braun E, Aronson D, Azzam ZS. Clinical and experimental pain perception is attenuated in patients with painless myocardial infarction. PAIN 2007;133:120-7.

${ }^{2}$ Sadigh-Lindell, B, Sylvén, C, Berglund, M, \& Eriksson, BE. Role of adenosine and opioid-receptor mechanisms for pain in patients with silent myocardial ischemia or angina pectoris: a double-blind, placebo-controlled study. J Cardiovasc Pharmacol 2003;42:757-763.

${ }^{3}$ Strigo IA, Duncan GH, Boivin M, Bushnell MC. Differentiation of visceral and cutaneous pain in the human brain. J Neurophysiol 2003;89:3294-303.

${ }^{4}$ Yarnitsky D, Granot M, Granovsky Y. Pain modulation profile and pain therapy: between pro- and antinociception. PAIN 2014;155:663-5. 